hep-th/9706225

\title{
EuClideAn SYM TheORIEs By TIME REDUCTION And Special Holonomy Manifolds
}

\author{
Matthias BlaU ${ }^{1}$ \\ LPTHE-ENSLAPP \\ ENS-Lyon, 46 Allée d'Italie \\ F-69364 Lyon CEDEX 07, France
}

\author{
George Thompson $^{2}$ \\ ICTP \\ P.O. Box 586 \\ 34014 Trieste, Italy
}

\begin{abstract}
Euclidean supersymmetric theories are obtained from Minkowskian theories by performing a reduction in the time direction. This procedure elucidates certain mysterious features of Zumino's $N=2$ model in four dimensions, provides manifestly hermitian Euclidean counterparts of all non-mimimal SYM theories, and is also applicable to supergravity theories. We reanalyse the twists of the $4 \mathrm{~d} N=2$ and $N=4$ models from this point of view. Other applications include SYM theories on special holonomy manifolds. In particular, we construct a twisted SYM theory on Kähler 3-folds and clarify the structure of SYM theory on hyper-Kähler 4-folds.
\end{abstract}

\footnotetext{
${ }^{1}$ e-mail: mblau@enslapp.ens-lyon.fr

${ }^{2}$ e-mail: thompson@ictp.trieste.it

${ }^{3}$ URA 14-36 du CNRS, associée à l'E.N.S. de Lyon, et à l'Université de Savoie
} 


\section{Contents}

\begin{tabular}{lll}
\hline & Introduction & 1
\end{tabular}

2 Euclidean SYM Theories from Time Reduction $\quad 2$

2.1 Zumino's Model from $N=1 d=(5+1) \mathrm{SYM}$. . . . . . . . . . . . . 3

2.2 R-Symmetries, Chiral Symmetries, and Twisting . . . . . . . . . . 5

2.3 General Properties . . . . . . . . . . . . . . . . . 7

2.4 Twisted $N=4$ Theories . . . . . . . . . . . . . . . . . . . . . . . 8

3 Euclidean SYM Theories on Special Holonomy Manifolds 10

3.1 General Aspects of Euclidean SYM Theories with Scalar Supercharges 11

3.2 SYM Theories on Kähler and Calabi-Yau Manifolds . . . . . . . . . 12

3.3 A Brief Survey of SYM Theories on Other Special Holonomy Manifolds 16

\begin{tabular}{|lr}
\hline A Notation & 19
\end{tabular}

\begin{tabular}{lr}
\hline References & 20
\end{tabular}

\section{INTRODUCTION}

This paper is concerned with a number of related issues. In one way or another they are all tied to the problem of finding Euclidean supersymmetric Yang-Mills (SYM) theories. The way we will produce these theories is by dimensional reduction of Minkowskian theories along some directions one of which is time. This construction leads to manifestly hermitian Euclidean SYM actions and thus to viable Euclidean counterparts of all non-minimal Minkowskian SYM theories. It reproduces the known Euclidean SYM actions, e.g. Zumino's $N=2$ supersymmertic instanton theory in $d=4$ [1], and explains in a natural way the features one has come to expect of such theories, such as non-compact R-symmetry groups and one scalar field whose kinetic term has the wrong sign (for a recent review of these matters see [2]).

One place where Euclidean SYM theories play an important role is in the context of topological field theories. We reanalyse the known twists of the $d=4 N=2$ [3] and $N=4$ [4, 5, 6, 7, 8, 9] theories by twisting directly the Euclidean SYM actions via the non-compact R-symmetry groups and 
show that the reality properties of the fields (which are usually introduced by hand) and of the action of the twisted theory are a consequence of the hermiticity of the underlying Euclidean SYM action.

Euclidean SYM actions also appear naturally in the context of D-brane instantons in string theory円 [0, 8], and for this and related reasons there has been some interest recently in (twisted) Euclidean SYM theories on $d>4$ manifolds with special holonomy groups [11, 12, 13]. These provide field theory realizations of the generalized self-duality conditions studied in [14, 15] and hence a topological field theory counterpart of the mathematical research programme outlined in [16]. Here we survey these constructions and attempt to clarify the relation between twisted theories and theories on manifolds possessing covariantly constant spinors. We point out the existence of a new twisted field theory on Kähler three-folds (modelling the Donaldson-Uhlenbeck-Yau equations), and describe the theory (and generalized self-duality condition) one obtains on hyper-Kähler eight-manifolds.

\section{Euclidean SyM Theories from Time Reduction}

Many years ago Zumino wrote down a Euclidean version of the $N=2 \mathrm{SYM}$ theory in four dimensions [1]. There are two rather striking features of this model. The first is that one of the two scalars (a true scalar) has a kinetic term with the wrong sign, whereas the other (the pseudo-scalar) has the standard sign. With the Minkowskian metric no such 'degenerate' behaviour is present. The second surprise is that in the Minkowskian theory there is a chiral symmetry that is compact while the Lorentz group $S O(3,1)$ is not whereas in the Euclidean theory the situation is reversed, the chiral symmetry group is non-compact while the Lorentz group $S O(4)$ is compact. These differences can be accomodated by starting with a single theory in 6 dimensions. The observation is quite simple and also has a straightforward generalization to other Euclidean SYM theories.

\footnotetext{
${ }^{1}$ In this context, the appearance of a scalar field with the 'wrong' sign alluded to above is natural as it should correspond to the time-like collective coordinate of the instanton, in the spirit of [10].
} 


\subsection{Zumino's Model From $N=1 d=(5+1)$ SYM}

Let us first describe the procedure in words. In $d=6$ one starts with the usual Minkowskian $N=1$ SYM theory. The field content of this theory consists of one six-vector (the gauge field) and one Weyl fermion in the $\mathbf{4}$ of $S O(5,1)$. Apart from the gauge group and the $N=1$ supersymmetry the model enjoys an $S U(2)_{\mathcal{R}}$ symmetry.

Now suppose that we dimensionally reduce in the standard way along two space directions. In this way we will obtain an $N=2$ theory with Minkowskian signature in four dimensions. The Lorentz group decomposes as $S O(5,1) \rightarrow S O(3,1) \times S O(2)$, where the $S O(2)$ is an internal symmetry group from the four dimensional stance. It is this $S O(2) \sim U(1)$ that plays the role of the compact chiral symmetry alluded to above. The two scalars that one gets from the six vector transform under the $S O(2)$ while the fourvector remains inert. The two scalars are naturally grouped into one complex scalar. In this way we have recovered all the features of the four-dimensional theory from the six-dimensional one.

Now let us perform a variant on this theme. We dimensionally reduce in one spatial direction and in the time direction. The resulting theory is a four dimensional Euclidean theory. There are two scalars here as well but one has the wrong sign (the one that is the time component of the gauge field). The Lorentz group now decomposes as $S O(5,1) \rightarrow S O(4) \times S O(1,1)$. The $S O(1,1)$ is now the non-compact chiral symmetry group. Once more the general features of Zumino's theory are reproduced.

One can perform the reductions explicitly and since we will need the fermionic action anyway we present the, perhaps unfamiliar, time reduction here. We begin in 6 dimensions. The spinor part of the $N=1$ theory is

$$
\int d^{6} x \bar{\psi}_{6} \not D \psi_{6}
$$

where $\psi$ is a Weyl spinor. A basis for the $\gamma$-matrices can be found in the Appendix. We 'compactify' (i.e. dimensinally reduce along) the time-direction $t=x_{0}$. Doing so one should pick up a Euclidean theory in 5 dimensions. In particular the spinor content should be that of a four dimensional Euclidean Dirac spinor, with the usual action. At first, however, the action (2.1), becomes

$$
\int d^{5} x \psi_{6}^{* T} \Gamma^{0}\left[\Gamma^{m} D_{m}+\Gamma^{0} A_{0}\right] \psi_{6}
$$


which has a seemingly spurious $\Gamma_{0}$ in it. But the spinor is chiral so we can write it as

$$
\psi_{6}=\left(\begin{array}{c}
\psi_{E} \\
0
\end{array}\right)
$$

and the action (2.2) becomes

$$
\int d^{5} x \psi_{E}^{* T}\left[\gamma^{m} D_{m}-A_{0}\right] \psi_{E}
$$

Upon further reduction along $x_{5}$ to 4 dimensions one obtains

$$
\int d^{4} x \psi_{E}^{* T}\left[\gamma^{\mu} D_{\mu}+\gamma_{5} A_{5}-A_{0}\right] \psi_{E} .
$$

Note that the field $A_{0}$ is the one that comes in with the wrong sign in the kinetic term, Zumino calls it $A$. The coupling we have found in (2.4) between the fermions and the (pseudo-) scalars $A_{5}$ and $A_{0}$ agrees with that given by Zumino [1]. One can easily check that the other parts of the action also agree.

We would now like to compare our dimensional reduction point of view with some recent work on Wick rotations [2]. These authors show that a consistent manner for obtaining Zumino's Euclidean theory from the Minkowski theory is to perform a Wick rotation. This rotation, it is claimed, should have the interpretation of being a complex Lorentz transformation in a would-be $(0,4)$-plane (with the above notation). The authors, though they give more arguments as to why their prescription should be thought of as a five dimensional Lorentz transformation, admit that there are some 'loose ends' which need to be fixed. From our higher-dimensional point of view we see that they are indeed morally correct.

One starts in 5-dimensional Minkowski space (and to conform with the notation in [2] we assume a space reduction of the 6-dimensional theory along $\left.x_{4}\right)$. The coordinates are thus $\left(t=x_{0}, x_{i}, x_{5}\right)$ with $i=1,2,3$. One may pass to the four dimensional Minkowski space by 'fixing' $x_{5}=0$. All the fields then have the functional dependence $\psi\left(t, x_{i}, 0\right)$. From the five dimensional perspective one can now perform a complex Lorentz boost in the $\left(t, x_{5}\right)$ plane mapping $\left(t, x_{5}\right)$ to $\left(i x_{5}, i t\right)$. For the fermions one finds

$$
\psi\left(t, x_{i}, 0\right) \rightarrow \mathrm{e}^{\frac{\pi}{2} i \Gamma_{0} \Gamma_{5}} \psi\left(0, x_{i}, i t\right)
$$

which is precisely the observation in [2]. As regards the gauge fields, from the point of view of four-dimensional Minkowski space, $A_{5}$ is a scalar. However, 
the rotation defined above exchanges $A_{0}$ with $A_{5}$ and, because of the $i$ factor, $A_{5}$ combines with $A_{i}$ to form a Euclidean four-vector. $A_{0}$ is now a scalar with the wrong sign for the kinetic term, exactly as in the above derivation of Zumino's model. Notice also that the pseudo-scalar, which was the space component $A_{4}$ of the gauge field, does not transform.

Of course the point of the paper [2] is to give a formulation of Euclidean supersymmetry even when there is no extended supersymmetry in the Minkowskian theory, and this certainly goes beyond what we are able to do here.

\subsection{R-Symmetries, Chiral Symmetries, and Twisting}

The $S U(2)_{\mathcal{R}}$ symmetry in $d=6$ is made up of a 'charge conjugation' symmetry

$$
\delta \psi_{6}=\mathcal{C} \psi_{6}^{*}
$$

(see the Appendix) as well as a normal phase transformation

$$
\delta \psi_{6}=i \psi_{6}
$$

and their commutator. Thus the single Weyl fermion (together with its complex conjugate) transforms as a doublet of $S U(2)_{\mathcal{R}}$. It can thus be thought of as a $(\mathbf{4}, \mathbf{2})$ of $S O(5,1) \times S U(2)_{\mathcal{R}}$ satisfying a symplectic MajoranaWeyl condition [17], which is possible since both representations are pseudoreal. This gives the four on-shell degrees of freedom required to match those of the gauge field.

This symmetry survives the dimensional reduction regardless of the signature that one chooses for the reduced space. Upon reduction to four Euclidean dimensions, the $\mathbf{4}$ of $S O(5,1)$ branches to the $(\mathbf{2}, \mathbf{1}) \oplus(\mathbf{1}, \mathbf{2})$ of $S O(4) \sim S U(2)_{L} \times S U(2)_{R}$. If we write

$$
\Psi_{L}=\left(\begin{array}{c}
\psi_{L} \\
\sigma_{2} \psi_{L}^{*}
\end{array}\right), \quad \Psi_{R}=\left(\begin{array}{c}
\psi_{R} \\
\sigma_{2} \psi_{R}^{*}
\end{array}\right)
$$

then the $S U(2)_{\mathcal{R}}$ symmetry takes the simple form

$$
\delta_{i} \Psi_{L . R}=\left(\sigma_{i} \otimes \mathbb{I}\right) \Psi_{L, R} .
$$

Note that while there are no Majorana spinors in four Euclidean dimensions, this being the obvious obstacle to formulating Euclidean $d=4$ SYM theories, 
the four-dimensional spinors one obtains in this way satisfy a symplectic Majorana condition: they are symplectic Majorana-Weyl with respect to this $S U(2)_{\mathcal{R}}$.

Note also that, because of the $\sigma_{2}$ in the definition of the $\psi_{L . R}$, they tranform essentially the same way under Lorentz transformations as under the R-symmetry. Thus the twisting is straightforward directly within the Euclidean setting and one obtains the familiar Grassmann odd field content

$$
\begin{aligned}
(2,1,2) \oplus(1,2,2) & \rightarrow(2,2) \oplus(3,1) \oplus(1,1) \\
& \simeq\left(\psi, \chi^{+}, \eta\right)
\end{aligned}
$$

and scalar supercharge of Donaldson theory [3].

The ghost number symmetry arises on dimensional reduction. The precise form of the symmetry group depends on whether one does a space-space or space-time reduction. With a space-space reduction one picks up an $S O(2)$ symmetry which translates into a $U(1)$ symmetry if we group the two scalar fields into one complex field $\phi$. The action is

$$
\int d^{4} x \phi \Delta \bar{\phi}
$$

which is clearly $U(1)$ invariant. On the other hand, performing a space-time reduction we would also get an action of the form (2.12) except that $\phi$ and $\bar{\phi}$ appearing there are two independent real fields. The ghost symmetry is now $S O(1,1)$ which is simply an invariance of the action under scaling $\phi \rightarrow \lambda \phi$ and $\bar{\phi} \rightarrow \lambda^{-1} \bar{\phi}$ for $\lambda \in \mathbb{R}^{*}$. For the fermions, in the reduction to Euclidean space, one finds that the $S O(1,1)$ transformations act as

$$
\delta \psi_{6}=\lambda \Sigma_{50} \psi_{6}=\lambda\left(\begin{array}{cc}
-\gamma_{5} & 0 \\
0 & \gamma_{5}
\end{array}\right) \psi_{6}
$$

(this being the Lorentz generator in the (50) direction) once more in agreement with [1].

Within the functional integral approach to Donaldson theory [3], the two scalar fields $\phi$ and $\bar{\phi}$ are nevertheless usually treated as complex conjugates (at the expense of hermiticity of the action), and it is then legitimate to regard the ghost number symmetry as being $U(1)$ instead of $S O(1,1)$, as is usually done.

Finally, note that on four-manifolds admitting covariantly constant spinors (for $d=4$ this singles out K3 and $T^{4}$ ), the untwisted Euclidean SYM theory 
possesses a supersymmetry precisely of the same type (scalar supercharges) as the twisted theories above. In that case (we would say that) no twist is required. In this sense we differ semantically from the presentation in [11]. We will have more to say about such theories (in particular in $d>4$ ) in section 3. Other possibilities (partially twisted topological theories) can arise when the four-manifold $M_{4}$ is not irreducible but rather a product $M_{4}=M_{3} \times S^{1}$ or $M_{4}=M_{2} \times M_{2}^{\prime}$ (see e.g. [18]).

\subsection{General Properties}

It should be clear that the method outlined above to obtain a hermitian Euclidean SYM theory in $d=4$, time reduction of the Minkowskian $N=1$ theory in $d=6$ to $d=4$, generalizes in a straightforward to other SYM theories: starting with a SYM theory in $(d+p+1)$-dimensional Minkowski space, one can obtain a hermitian Euclidean SYM action in $d$ Euclidean dimensions by simply reducing along $p$ space-like and the one time-like direction. This is the Euclidean counterpart of the Minkowskian SYM theory obtained in the standard way by dimensional reduction along $(p+1)$ space-like directions. The Euclidean theory obtained in this way will have a non-compact internal R-symmetry group $S O(p, 1)$ and, as before, there will be fields (among them the time-component of the gauge field) having a kinetic term with the wrong sign.

Although in the above we have described the procedure for SYM theories. it clearly works equally well for other supersymmetric theories such as $N=1$ supergravity in $d=(10+1)$ which can be reduced to a Euclidean supergravity theory in $d=10$, a Euclidean analogue of type IIA supergravity.

For concreteness, however, and because it is of the most interest in the current endeavour to understand higher-dimensional SYM theories and the world-volume actions of D-branes, let us consider the $N=1$ SYM theory in $d=9+1$ dimensions. Its field content consists of a gauge field and a Majorana-Weyl fermion in the $\mathbf{1 6}$ of $S O(9,1)$. As a consequence there is no R-symmetry group in ten dimensions.

Standard dimensional reduction leads to Minkowskian SYM theories with 16 supercharges in $d<10$ with compact R-symmetry group $S O(10-d)$ arising form the branching

$$
S O(9,1) \rightarrow S O(d-1,1) \times S O(10-d) .
$$

The most prominent member of this hierarchy of theories is the scale in- 
variant and presumably S-dual $N=4$ theory in $d=3+1$ with R-symmetry group $S O(6) \sim S U(4)$.

By following the above procedure, (reduction from $(9+1)$ to $d$ Euclidean dimensions), one obtains Euclidean counterparts of these theories for all $d<10$. The relevant branching this time is

$$
S O(9,1) \rightarrow S O(d) \times S O(9-d, 1)
$$

exhibiting $S O(9-d, 1)$ as the non-compact internal R-symmetry group of the theory.

Under this branching, the vector representation 10 decomposes in the obvious way as

$$
\mathbf{1 0} \rightarrow(\mathbf{d}, \mathbf{1})+(\mathbf{1},(\mathbf{1 0}-\mathbf{d})) .
$$

The corresponding branchings of the $\mathbf{1 6}$ of $S O(9,1)$, the Majorana-Weyl spinor, can e.g. be read off from the table given by Seiberg in 19] (by reversing one of the columns). Subscripts $r$ and $p$ indicate real and pseudoreal representations.

\begin{tabular}{c|ccc} 
& $S O(9,1)$ & $\rightarrow$ & $S O(d) \times S O(9-d, 1)$ \\
\hline$d=9$ & $\mathbf{1 6}_{\mathbf{r}}$ & $\rightarrow$ & $\mathbf{1 6}_{\mathbf{r}}$ \\
$d=8$ & $\mathbf{1 6}_{\mathbf{r}}$ & $\rightarrow$ & $\left(\mathbf{8}_{\mathbf{s}, \mathbf{r}},+\mathbf{1}_{\mathbf{r}}\right)+\left(\mathbf{8}_{\mathbf{c}, \mathbf{r}},-\mathbf{1}_{\mathbf{r}}\right)$ \\
$d=7$ & $\mathbf{1 6}_{\mathbf{r}}$ & $\rightarrow$ & $\left(\mathbf{8}_{\mathbf{r}}, \mathbf{2}_{\mathbf{r}}\right)$ \\
$d=6$ & $\mathbf{1 6}_{\mathbf{r}}$ & $\rightarrow$ & $(\mathbf{4}, \mathbf{2})+(\overline{\mathbf{4}}, \overline{\mathbf{2}})$ \\
$d=5$ & $\mathbf{1 6}_{\mathbf{r}}$ & $\rightarrow$ & $\left(\mathbf{4}_{\mathbf{p}}, \mathbf{4}_{\mathbf{p}}\right)$ \\
$d=4$ & $\mathbf{1 6}_{\mathbf{r}}$ & $\rightarrow$ & $\left(\mathbf{2}_{\mathbf{p}}, \mathbf{4}_{\mathbf{p}}\right)+\left(\mathbf{2}_{\mathbf{p}}^{\prime}, \mathbf{4}_{\mathbf{p}}^{\prime}\right)$ \\
$d=3$ & $\mathbf{1 6}_{\mathbf{r}}$ & $\rightarrow$ & $\left(\mathbf{2}_{\mathbf{p}}, \mathbf{8}_{\mathbf{p}}\right)$ \\
$d=2$ & $\mathbf{1 6}_{\mathbf{r}}$ & $\rightarrow$ & $\left(+\mathbf{1}, \mathbf{8}_{\mathbf{s}}\right)+\left(-\mathbf{1}=\overline{+\mathbf{1}}, \mathbf{8}_{\mathbf{c}}=\overline{\mathbf{8}_{\mathbf{s}}}\right)$
\end{tabular}

Note that all the representations appearing on the right-hand side manifestly have real structures. For $d=7,8,9$ the representations are simply real and the spinors Majorana (-Weyl), for $d=3,4,5$ one has symplectic Majorana (-Weyl) spinors, and for $d=2,6$ the representaions are of the form of a complex representation plus its complex conjugate and thus also have an obvious real structure.

\subsection{Twisted $N=4$ Theories}

Among the SYM theories with 16 supercharges, the $d=4 N=4$ theory is of particular interest, in particular because of its suspected S-duality and 
its relation to toroidal compactifications of heterotic strings. Twisted $N=4$ theories were first investigated in [4] and then subsequently in [5, 6, 8, 9]. It was found that in general there are three inequivalent topological twists of the $N=4$ theory which we will refer to as the half-twisted model [4, 9], the A-model [5, 20, 21, 8, 9] and the B-model [6, 8, 9].

As reviewed e.g. in [9], all these investigations were based on a (from our point of view) hybrid approach in which one uses the compact R-symmetry group $S O(6) \sim S U(4)$ of the Minkowskian theory to twist the Lorentz gorup $S O(4) \sim S O(3)_{L} \times S O(3)_{R}$ of the Euclidean theory. One is thus (implicitly) complexifying the theory and reality conditions on the fields have to be reimposed by hand at the end. From this point of view, the three $N=4$ twists are most conveniently described by the branchings

$$
\begin{aligned}
& S O(6) \rightarrow S O(4) \times S O(2) \sim S O(3)_{1} \times S O(3)_{2} \times S O(2) \\
& S O(6) \rightarrow S O(3)_{A} \times S O(3)_{B} .
\end{aligned}
$$

For the half-twisted model one twists $S O(3)_{L}$ by $S O(3)_{1}$ (i.e. replaces $S O(3)_{L}$ by $\operatorname{diag}\left(S O(3)_{L} \times S O(3)_{1}\right)$, for the B-model one furthermore twists $S O(3)_{R}$ by $S O(3)_{2}$ and for the A-model one twists $S O(3)_{L}$ by, say, $S O(3)_{A}$.

As we will now show (and as might have been expected) we can completely side-step this problem of compelexification and reality conditions by working directly with the hermitian Euclidean SYM action and its non-compact Rsymmetry group $S O(5,1)$. The field content is

$$
\begin{array}{ll}
d=4 & \mathbf{1 0} \rightarrow(\mathbf{2}, \mathbf{2} ; \mathbf{1})+(\mathbf{1}, \mathbf{1} ; \mathbf{6}) \\
& \mathbf{1 6} \rightarrow(\mathbf{2}, \mathbf{1} ; \mathbf{4})+(\mathbf{1}, \mathbf{2} ; \overline{\mathbf{4}})
\end{array}
$$

The two relevant branchings of the R-symmetry group $S O(5,1)$ are the regular branching $S O(5,1) \rightarrow S O(3)_{1} \times S O(3)_{2} \times S O(1,1)$,

$$
\begin{aligned}
& 4 \rightarrow(2,1)^{+1}+(1,2)^{-1} \\
& \overline{4} \rightarrow(2,1)^{-1}+(1,2)^{+1} \\
& 6 \rightarrow(1,1)^{2}+(1,1)^{-2}+(2,2)^{0}
\end{aligned}
$$

and the branching $S O(5,1) \rightarrow S O(3) \times S O(2,1)$,

$$
\begin{aligned}
& \mathbf{4} \rightarrow(2,2) \\
& \overline{4} \rightarrow(2,2) \\
& 6 \rightarrow(1,3)+(3,1)
\end{aligned}
$$


The different twists can now be obtained as follows. Using the first branching and twisting $S O(3)_{L}$ by $S O(3)_{1}$ one obtains the half-twisted model with $N_{T}=1$ (meaning one topological or scalar supercharge), residual global symmetry group $S O(3)_{2} \times S O(1,1)$, and with field content

$$
\begin{aligned}
\text { L1-Twist } \Rightarrow & \mathbf{1 0} \rightarrow(2,2 ; \mathbf{1})^{\mathbf{0}}+(\mathbf{1}, \mathbf{1} ; \mathbf{1})^{+2,-2}+(\mathbf{2}, \mathbf{1} ; \mathbf{2})^{\mathbf{0}} \\
\mathbf{1 6} & \rightarrow(\mathbf{1}+\mathbf{3}, \mathbf{1} ; \mathbf{1})^{+\mathbf{1}}+(\mathbf{2}, \mathbf{2} ; \mathbf{1})^{-\mathbf{1}}+(\mathbf{2}, \mathbf{1} ; \mathbf{2})^{-\mathbf{1}}+(\mathbf{1}, \mathbf{2} ; \mathbf{2})^{+1}
\end{aligned}
$$

Twisting also $S O(3)_{R}$ by $S O(3)_{2}$, one obtains the B-model with $N_{T}=2$, residual global symmetry $S O(1,1)$ and field content

$$
\begin{aligned}
& \text { L1-R2-Twist } \Rightarrow \mathbf{1 0} \rightarrow(\mathbf{2}, \mathbf{2})^{\mathbf{0}}+(\mathbf{1}, \mathbf{1})^{+\mathbf{2},-2}+(\mathbf{2}, \mathbf{2})^{\mathbf{0}} \\
& 16 \rightarrow 2 \times(\mathbf{1}, \mathbf{1})^{+1}+(3,1)^{+1}+(1,3)^{+1}+2 \times(2,2)^{-1}
\end{aligned}
$$

To obtain the A-model one uses the second branching to twist the $S O(3)_{L}$ by $S O(3)$. Then $S O(2,1)$ remains as a global symmetry group and the field content is

$$
\begin{aligned}
& \text { A-Twist } \Rightarrow \mathbf{1 0} \rightarrow(\mathbf{2}, \mathbf{2} ; \mathbf{1})+(\mathbf{1}, \mathbf{1} ; \mathbf{3})+(\mathbf{3}, \mathbf{1} ; \mathbf{1}) \\
& 16 \rightarrow(1,1 ; 2)+(3,1 ; 2)+(2,2 ; 2)
\end{aligned}
$$

Note that the fermionic field content is simply an $S L(2, \mathbb{R})$ doublet of that of Donaldson theory. In particular, the reality properties of the fields inherited from the hermitian Euclidean action are $S L(2, \mathbb{R})$-invariant. This explains why in [4, 9] it was found necessary to break the (hybrid) R-symmetry group $S O(3)_{B}$, obtained from the branching (2.18), down to $S O(2)$ to obtain a real action.

\section{Euclidean SYM Theories on Special Holonomy Mani- FOLDS}

In this section, we will deal with Euclidean SYM theories on $d>4$ manifolds. At present the field theoretic status of SYM theories in $d>4$ is not entirely clear (for a recent discussion see [19]). However, it appears to be reasonable to believe that whenever the theory admits a scalar (i.e. singlet) supercharge its associated topological field theory, defined by the BRST cohomology of this supercharge, is meaningful and can be studied in its own right. While this certainly requires further justification, we will proceed by optimistically adopting this as a working hypothesis. 
Once again, for concreteness and because we are primarily interested in $d>4$, we will focus on the SYM theories with 16 supercharges obtained by dimensional reduction of the $N=1$ theory in $d=(9+1)$.

\subsection{General Aspects of Euclidean SYM Theories with Scalar SUPERCHARGES}

As is well known (and we have recalled in section 2), one way to produce a SYM theory with scalar supercharges is to twist, i.e. to use the R-symmetry group to find a new embedding of the space (-time) rotation group into the global symmetry group, in such a way that at least one of the supercharges of the untwisted theory becomes a singlet with respect to the new rotation group. Now generically the rotation group (structure group of the tangent bundle) of a $d$-manifold is $S O(d)$ while the R-symmetry group is $S O(9-d, 1)$. Thus for $d>4$ the R-symmetry group is simply not large enough to permit a twisting. 20 One is thus led to consider manifolds with reduced holonomy (structure) groups $G_{M} \subset S O(d)$. For a recent survey of what is known about holonomy groups, see e.g. [22].

Essentially the only interesting possibilities (of potentially 'twistable' holonomy groups not admitting covariantly constant spinors) are Kähler $n$-folds with holonomy group $G_{M}=U(n) \subset S O(2 n)$. The underlying reason for why twisting works in this case is that on a Kähler $n$-fold $\left(\operatorname{spin}_{c}\right)$ spinors can be identified with twisted differential forms (more precisely one has

$$
\left(\mathbb{S}^{+} \oplus \mathbb{S}^{-}\right) \otimes K_{M}^{+1 / 2} \simeq \Omega_{M}^{*, 0}
$$

where $K_{M}$ is the canonical bundle of $M$ and $\mathbb{S}^{ \pm}$are the spin-bundles) and that one can use $U(1)$ subgroups of the R-symmetry group to untwist them, i.e. to mimic tensoring by square roots of $K_{M}$ (see also [23]). We will explain this in somewhat more detail below.

Another way to produce a SYM theory with scalar supercharges is to place it on a spin-manifold $M$ admitting covariantly constant spinors. This means that one is once again led to consider reduced holonomy groups $G_{M} \subset$ $S O(d)$, this time such that one obtains at least one $G_{M}$-singlet from the branching of the spinor representation under $S O(d) \rightarrow G_{M}$. This is of course a well known property of Calabi-Yau manifolds (and the principal

\footnotetext{
${ }^{2}$ With the possible exception of $d=5$ if one closes one's eyes to the difference between $S O(5)$ and $S O(4,1)$.
} 
reason for why they appear in the context of string compactifications in the first place). In $d<10$, the only other non-trivial possibilities are known to be K3-surfaces (i.e. Calabi-Yau or hyper-Kähler 2-folds with holonomy $S U(2)$ ), hyper-Kähler 4-folds with holonomy group $S p(2)$, and the exceptional $G_{2}$ and $\operatorname{Spin}(7)$ holonomy Joyce seven- and eight-manifolds.

Typically, on such a manifold the spinor bundle becomes isomorphic to a tensor bundle, as exemplified by the branching

$$
S O(7) \rightarrow G_{2}: \quad \mathbf{8} \rightarrow \mathbf{1}+\mathbf{7}
$$

which means that a spinor on a $G_{2}$-manifold can be regarded as a pair $\left(\eta, \psi_{\mu}\right)$ consisting of a scalar and a vector field. Thus a SYM theory on such a manifold acquires all (or at least many of) the characteristics of a cohomological topological field theory all by itself, i.e. without twisting it explicitly. Some aspects of these theories (notably for $G_{M}=S U(4)$ and $\left.G_{M}=\operatorname{Spin}(7)\right)$ have been studied in [11] (see also [12]), where the relation to the generalized self-duality conditions of 114, 15] and the moduli problems studied in [16] were pointed out. We will survey the possibilities that arise in this way below in the light of the considerations of section 2 .

Among the admissible metric holonomy groups there are also pseudo-versions of the groups above which can occur as reductions of pseudo-Riemannian manifolds [22], such as $U(p, q) \subset S O(2 p, 2 q)$ and a non-compact counterpart of the reduction (3.2), namely $G_{2(1)} \subset S O(4,3)$. If interest in exotic signatures persists in the physics literature, then perhaps these manifolds will also come to play a role.

\subsection{SYM Theories on Kähler and Calabi-Yau Manifolds}

In order to determine the spectrum (and possible twists) of SYM theories on Kähler and Calabi-Yau $n$-folds, we require the branching of the vector and spinor representations under $S O(2 n) \rightarrow U(n)$. As on a Kähler manifold a one-form can be decomposed as the sum of a $(1,0)$ and a $(0,1)$ form, the general structure for the decomposition of the fundamental representation $\mathbf{2 n}$ of $S O(2 n)$ under the branching $S O(2 n) \rightarrow U(n)$ is

$$
\mathbf{2 n} \rightarrow \mathbf{n}^{+\mathbf{q}}+\overline{\mathbf{n}}^{-\mathbf{q}} .
$$

Here $\mathbf{n}$ and $\overline{\mathbf{n}}$ designate representations of $S U(n)$ and $q$ is a $U(1)$-charge. It is conventionally normalized to be the smallest integers such that all 
representations have integer $U(1)$-charge. For $n=2,3,4$ one has $q=1,2,1$ respectively. Thus, as $\mathbf{n}^{+\mathbf{q}}$ corresponds to $(1,0)$-forms, the canonical line bundle $K$ and its square root $K^{1 / 2}$ then correspond to the representations $\mathbf{1}^{+\mathbf{n q}}$ and $\mathbf{1}^{+\mathbf{n q} / \mathbf{2}}$ respectively.

To simplify the notation, occasionally when one $S U(n)$ representations $\mathbf{d}$ appears with several $U(1)$ charges $a, b, c, \ldots$, we will group them together and write them as $\mathbf{d}^{\mathbf{a}, \mathbf{b}, \mathbf{c}, \ldots}$. With these notations, one then has the following table for the relevant (vector, spinor, two-form) representations:

$$
\begin{aligned}
& S O(4) \rightarrow U(2) \quad 4=(\mathbf{2}, \mathbf{2}) \quad \rightarrow \quad \mathbf{2}^{+\mathbf{1}}+\mathbf{2}^{-\mathbf{1}} \\
& \mathbf{2}_{\mathrm{L}}=(\mathbf{2}, \mathbf{1}) \quad \rightarrow \quad \mathbf{2}^{\mathbf{0}} \\
& \mathbf{2}_{\mathrm{R}}=(\mathbf{1}, \mathbf{2}) \quad \rightarrow \quad \mathbf{1}^{+\mathbf{1}}+\mathbf{1}^{-1} \\
& \mathbf{6}=(\mathbf{3}, \mathbf{1})+(\mathbf{1}, \mathbf{3}) \rightarrow \mathbf{3}^{\mathbf{0}}+\mathbf{1}^{+\mathbf{2}, \mathbf{0},-\mathbf{2}} \\
& S O(6) \rightarrow U(3) \quad 6 \quad \rightarrow \quad \mathbf{3}^{\mathbf{2}}+\overline{\mathbf{3}}^{-\mathbf{2}} \\
& 4 \quad \rightarrow \quad \mathbf{1}^{+3}+3^{-1} \\
& \overline{4} \quad \rightarrow \quad \mathbf{1}^{-3}+\overline{3}^{+1} \\
& 15 \rightarrow 1^{0}+3^{-4}+\overline{3}^{+4}+8^{0} \\
& S O(8) \rightarrow U(4) \quad \mathbf{8}_{\mathbf{v}} \quad \rightarrow \quad \mathbf{4}^{+\mathbf{1}}+\overline{\mathbf{4}}^{-\mathbf{1}} \\
& 8_{\mathrm{S}} \quad \rightarrow \quad \mathbf{1}^{+2,-2}+6^{0} \\
& 8_{\mathrm{c}} \quad \rightarrow \quad 4^{-1}+\overline{4}^{+1} \\
& 28 \quad \rightarrow \quad 1^{0}+6^{+2,-2}+15^{0}
\end{aligned}
$$

Here for $S O(4)$ we have assumed that the reduction to $U(2)$ proceeds via $S U(2)_{L} \times S U(2)_{R} \rightarrow S U(2)_{L} \times U(1)_{R}$. Note that in each case, the decomposition of the two-forms contains a singlet $\mathbf{1}^{\mathbf{0}}$. This corresponds to the covariantly constant Kähler two-form. The other terms in the two-form decomposition correspond to the $(2,0)$ and $(0,2)$ forms and to the $(1,1)$-forms not proportional to the Kähler form. The latter transform in the adjoint of $S U(n)$, as follows from $\mathbf{n} \times \overline{\mathbf{n}}=\mathbf{1}+\left(\mathbf{n}^{2}-\mathbf{1}\right)$. Upon restriction to CalabiYau $n$-folds with holonomy group $S U(n)$, one finds two covariantly constant spinors of the same chirality for $n=2,4$, and two of opposite chirality for $n=3$.

It follows from the above that on a Kähler $n$-fold for $n=2,3,4$ one has the following relation between differential forms and twisted $\operatorname{spinors}\left(\operatorname{spin}_{c}\right.$ 
spinors - these exist on any Kähler manifold $M$ even when $M$ is not spin):

$$
\begin{array}{lll}
n=2 & \mathbb{S}^{+} \otimes K^{+1 / 2} \simeq \mathbf{1}^{\mathbf{0}}+\mathbf{1}^{+\mathbf{2}} & =\Omega^{\text {even }, 0} \\
& \mathbb{S}^{+} \otimes K^{-1 / 2} \simeq \mathbf{1}^{\mathbf{0}}+\mathbf{1}^{-\mathbf{2}} & =\Omega^{0, \text { even }} \\
& \mathbb{S}^{-} \otimes K^{+1 / 2} \simeq \mathbf{2}^{+\mathbf{1}} & =\Omega^{\text {odd }, 0} \\
& \mathbb{S}^{-} \otimes K^{-1 / 2} \simeq \mathbf{2}^{-\mathbf{1}} & =\Omega^{0, \text { odd }} \\
n=3 & \mathbb{S}^{+} \otimes K^{+1 / 2} \simeq \mathbf{1}^{+\mathbf{6}}+\mathbf{3}^{+\mathbf{2}} & =\Omega^{\text {odd }, 0} \\
& \mathbb{S}^{+} \otimes K^{-1 / 2} \simeq \mathbf{1}^{\mathbf{0}}+\mathbf{3}^{-\mathbf{4}} & =\Omega^{0, \text { even }} \\
& \mathbb{S}^{-} \otimes K^{+1 / 2} \simeq \mathbf{1}^{\mathbf{0}}+\overline{\mathbf{3}}^{+\mathbf{4}} & =\Omega^{\text {even }, 0} \\
& \mathbb{S}^{-} \otimes K^{-1 / 2} \simeq \mathbf{1}^{-\mathbf{6}}+\overline{\mathbf{3}}^{-\mathbf{2}} & =\Omega^{0, \text { odd }} \\
n=4 & \mathbb{S}^{+} \otimes K^{+1 / 2} \simeq \mathbf{1}^{+\mathbf{4}}+\mathbf{1}^{\mathbf{0}}+\mathbf{6}^{\mathbf{2}} & =\Omega^{\text {even }, 0} \\
& \mathbb{S}^{+} \otimes K^{-1 / 2} \simeq \mathbf{1}^{\mathbf{0}}+\mathbf{1}^{-\mathbf{4}}+\mathbf{6}^{-\mathbf{2}} & =\Omega^{0, \text { even }} \\
& \mathbb{S}^{-} \otimes K^{+1 / 2} \simeq \mathbf{4}^{+\mathbf{1}}+\overline{\mathbf{4}}^{+\mathbf{3}} & =\Omega^{\text {odd }, 0} \\
& \mathbb{S}^{-} \otimes K^{-1 / 2} \simeq \mathbf{4}^{-\mathbf{3}}+\overline{\mathbf{4}}^{-\mathbf{1}} & =\Omega^{0, \text { odd }}
\end{array}
$$

On a Calabi-Yau manifold, $K$ is trivial, and choosing the trivial square root of $K$, the above twist is trivial. Thus on a Calabi-Yau manifold, one can identify spinors directly with differntial forms.

The above correspondence, valid at the level of complex representations, does not yet take into account the effect of being able to impose reality (Majorana) conditions on the spinors in certain cases (notably for $n=4$ ). These reality properties can be read off from table (2.17), and combining this with (3.4) one finds that the $U(n) \times S O(9-2 n, 1)$ field content of the $N=1 d=(9+1)$ SYM theory reduced to a Kähler $n$-fold is

$$
\begin{array}{ll}
n=2 \quad \mathbf{1 0} \rightarrow\left(\mathbf{2}^{+\mathbf{1}}+\mathbf{2}^{-\mathbf{1}} ; \mathbf{1}\right)+\left(\mathbf{1}^{\mathbf{0}} ; \mathbf{6}\right) \\
\mathbf{1 6} \rightarrow\left(\mathbf{2}^{\mathbf{0}} ; \mathbf{4}\right)+\left(\mathbf{1}^{+}+\mathbf{1}^{-\mathbf{1}} ; \mathbf{4}^{\prime}\right) \\
n=3 \quad \mathbf{1 0} \rightarrow\left(\mathbf{3}^{+\mathbf{2}}+\overline{\mathbf{3}}^{-\mathbf{2}} ; \mathbf{1}\right)+\left(\mathbf{1}^{\mathbf{0}} ; \mathbf{4}\right) \\
& \mathbf{1 6} \rightarrow\left(\mathbf{1}^{+\mathbf{3}}+\mathbf{3}^{-\mathbf{1}} ; \mathbf{2}\right)+\left(\mathbf{1}^{-\mathbf{3}}+\overline{\mathbf{3}}^{+\mathbf{1}} ; \overline{\mathbf{2}}\right) \\
n=4 & \mathbf{1 0} \rightarrow \mathbf{4}^{+\mathbf{1} ; \mathbf{0}}+\overline{\mathbf{4}}^{-\mathbf{1} ; \mathbf{0}}+\mathbf{1}^{\mathbf{0} ;+\mathbf{2}}+\mathbf{1}^{\mathbf{0} ;-\mathbf{2}} \\
& \mathbf{1 6} \rightarrow \mathbf{4}^{-\mathbf{1} ;+\mathbf{1}}+\overline{\mathbf{4}}^{+\mathbf{1} ;+\mathbf{1}}+\mathbf{6}^{\mathbf{0} ;-\mathbf{1}}+\mathbf{1}^{+\mathbf{2} ;-\mathbf{1}}+\mathbf{1}^{-\mathbf{2} ;-\mathbf{1}}
\end{array}
$$

In a number of respects, the case $n=2$ is somewhat special. First of all, one does not need a reduced holonomy group in order to be able to twist the theory. Secondly, these three standard twists are well understood and can readily be specialized to Kähler manifolds if this is required (see e.g. [5]). And finally, as is also well known, on a hyper-Kähler (K3) 2-fold, one does not need to twist at all and one obtains directly an $N_{T}=8$ theory. This is consistent with the fact that a K3 is known to preserve $1 / 2$ of the 
sueprsymemtries, thus reducing the 16 supercharges of the flat space theory to 8 .

For $n=3$, the R-symmetry group is $S O(3,1) \sim S L(2, \mathbb{C})$ and in this case the theory can be twisted via a suitable $U(1)$-subgroup of $S L(2, \mathbb{C})$ (chosen such as to cancel the $U(1)$-charges of the $S U(3)$ singlets appearing in the spinor branching). Concretely, with a convenient normalization of the $U(1)$ charges, one has

$$
\begin{array}{rcccc}
\mathbf{1 6} & \rightarrow \quad\left(\mathbf{1}^{+\mathbf{3}}+\mathbf{3}^{-\mathbf{1}}, \mathbf{2}\right)+\left(\mathbf{1}^{-\mathbf{3}}+\overline{\mathbf{3}}^{+\mathbf{1}}, \overline{\mathbf{2}}\right) & \text { of } & U(3) \times S L(2, \mathbb{C}) \\
\rightarrow & \left(\mathbf{1}^{+\mathbf{3}}+\mathbf{3}^{-\mathbf{1}}, \mathbf{1}^{ \pm \mathbf{3}}\right)+\left(\mathbf{1}^{-\mathbf{3}}+\overline{\mathbf{3}}^{+\mathbf{1}}, \mathbf{1}^{ \pm \mathbf{3}}\right) & \text { of } & U(3) \times U(1) \\
\rightarrow & \mathbf{1}^{+\mathbf{6}, \mathbf{0}, \mathbf{0},-\mathbf{6}}+\mathbf{3}^{+\mathbf{2}}+\mathbf{3}^{-\mathbf{4}}+\overline{\mathbf{3}}^{+\mathbf{4}}+\overline{\mathbf{3}}^{-\mathbf{2}} & \text { of } & U(3) \text { by twisting } \\
& \Omega^{*, 0} \oplus \Omega^{0, *} & &
\end{array}
$$

Thus this theory has $N_{T}=2$ and a residual global $\mathbb{C}^{*}$ symmetry, the centralizer of $U(1) \subset S L(2, \mathbb{C})$. The reality conditions on the spnors imply the obvious reality condition $\left(\omega^{p, 0}\right)^{*}=\omega^{0, p}$.

If one introduces the complex Grassmann odd fields $\eta^{0,0}, \psi^{1,0}, \chi^{2,0}, \eta^{3,0}$ and their complex conjugates, then the fermionic action can schematically be written as

$$
S_{F}=\int \eta^{0,3} \partial_{A} \chi^{2,0}+\omega^{2} \psi^{0,1} \partial_{A}^{*} \chi^{2,0}+\omega^{3} \eta^{0,0} \partial_{A}^{*} \psi^{1,0}+\text { c.c. }
$$

Upon twisting, the bosonic spectrum consists of the gauge field, two scalars, a $(3,0)$ and a $(0,3)$ form, and the action provides a (topological) field theory description of the DUY (Donaldson, Uhlenbeck, Yau) equations

$$
\begin{gathered}
F^{2,0}=F^{0,2}=0 \\
\omega^{n-1} \wedge F^{1,1}=0
\end{gathered}
$$

for $n=3$, describing stable vector bundles. Its one-loop approximation can be described by the generalized bc-systems of [23] and is thus governed by the Ray-Singer torsion of $M$. If the 3 -fold is Calabi-Yau, then once again no twisting is necessary, the theory exhibits an $N_{T}=4=16 / 4$ topological symmetry (and the full $S L(2, \mathbb{C})$ R-symmetry) and coincides with the theory studied in [11.

Finally, for $n=4$, the R-symmetry group is $S O(1,1)$ and hence cannot be used directly (i.e. while preserving the reality conditions) to twist the $U(1)$-subgroup of $U(4)$. On a Calabi-Yau 4-fold, however, one finds the 
$H$-theory of [11], an $N_{T}=2$ theory describing a holomorphic analogue [16] of Donaldson theory, i.e. gauge fields satisfying the holomorphic self-duality condition

$$
\star F^{0,2}=F^{0,2},
$$

where $\star: \Omega^{0,2} \rightarrow \Omega^{0,2}$ satifying $\star^{2}=1$ is defined via the holomorphic 4 -form. This self-duality condition arises as a consequence of the reality property of the $6=(4 \wedge 4)$ appearing in the branching of the $\mathbf{8}_{\mathrm{s}}$.

\subsection{A Brief Survey of SyM Theories on Other Special Holon- OMY MANIFOLDS}

Apart from Kähler and Calabi-Yau manifolds, discussed above, the only other manifolds on which SYM theories acquire scalar supercharges are $\operatorname{Spin}(7)$ (or octonionic) and hyper-Kähler eight-manifolds and $G_{2}$ sevenmanifolds. The first case has been discussed at length in [11, 12] to which we refer for details. Here the moduli space in question is defined by the equation 14

$$
F_{8 i}=c_{i j k} F_{j k}
$$

where the $c_{i j k}$ are the octonionic structure constants. This equation can be understood more invariantly from the branching $\boldsymbol{8}_{\mathrm{s}} \rightarrow \mathbf{7}+\mathbf{1}$ which exhibits the one covariantly constant spinor $\zeta\left(\mathbf{8}_{\mathbf{v}}\right.$ and $\mathbf{8}_{\mathbf{c}}$ remain irreducible under $S O(8) \rightarrow S O(7))$. Consequently the two-form decomposition is $\mathbf{2 8} \rightarrow \mathbf{2 1}+\mathbf{7}$, corresponding to the two eigenspaces of the operator

$$
T^{i j k l}=\zeta^{T} \gamma^{[i} \gamma^{j} \gamma^{k} \gamma^{l]} \zeta
$$

on two-forms, and the seven equations (3.11) represent a projection onto the 21. As anticipated in [11, 12], the field theory associated to this moduli problem arises from the eight-dimensional Euclidean SYM theory,

The $G_{2}$ case arises from a straightforward dimensional reduction of the octonionic theory: the branching (3.2) induces the two-form decomposition $\mathbf{2 1} \rightarrow \mathbf{1 4}+\mathbf{7}$ and the reduction of (3.11) projects onto the $\mathbf{1 4}$ [14].

Finally, we study the hyper-Kähler case in a little more detail. Let us initially consider the branching $S O(8) \rightarrow S p(2) \times S p(1)$ (so this would correspond to a quaternionic Kähler manifold). The vector, spinor, and two-form representations branch to

$$
8 \mathrm{v} \rightarrow(4,2)
$$




$$
\begin{aligned}
& \mathbf{8}_{\mathrm{s}} \rightarrow(\mathbf{5}, \mathbf{1})+(\mathbf{1}, \mathbf{3}) \\
& \mathbf{8}_{\mathrm{c}} \rightarrow(\mathbf{4}, \mathbf{2}) \\
& \mathbf{2 8} \rightarrow(\mathbf{1}, \mathbf{3})+(\mathbf{1 0}, \mathbf{1})+(\mathbf{5}, \mathbf{3})
\end{aligned}
$$

Thus a hyper-Kähler eight-manifold admits three covariantly constant spinors (of the same chirality). The defining representation of $S p(n)$ on $\mathbb{H}^{n}$ can be regarded as a representation $\mathbf{2 n}$ on $\mathbb{C}^{2 n}$. The holonomy group of an eightmanifold being reduced to $S p(2) \times S p(1)$ means that the (co-)vector $V_{\mu}$ can be written as $V_{a A}$ where $a=1,2,3,4$ and $A=1,2$. In other words, $\mathbf{8}_{\mathbf{v}} \rightarrow(\mathbf{4}, \mathbf{2})$. The reality condition $V_{\mu}^{*}=V_{\mu}$ can be written as

$$
V^{*, a A} \equiv V_{a A}^{*}=\epsilon^{a b} \epsilon^{A B} V_{b B}
$$

(4 and $\mathbf{2}$ being pseudo-real, $(\mathbf{4}, \mathbf{2})$ has a real structure). As $\mathbf{8}_{\mathrm{c}}$ branches in the same way as $\mathbf{8}_{\mathbf{v}}$, this also explains the real structure induced by $\mathbf{8}_{\mathbf{c}}$.

The $\mathbf{8}_{\mathbf{s}}$, on the other hand, branches to $(\mathbf{5}, \mathbf{1})+(\mathbf{1}, \mathbf{3})$. The second term corresponds to a real symmetric tensor $\eta_{(A B)}$ in the $\mathbf{2} S \mathbf{2}=\mathbf{3}$ of $S p(1)=$ $S U(2)$. The $\mathbf{5}$ of $S p(2)$ has the following interpretation. The $\wedge^{2} \mathbf{4}$ of $S p(2)$ is a six-dimensional representation which is, however, reducible as $S p(2)$ leaves invariant the symplectic form $\epsilon_{a b}$. Thus one has the decomposition

$$
\begin{aligned}
\mathbf{4} \wedge \mathbf{4} & =\mathbf{1}+\mathbf{5} \\
\omega_{a b} & =\epsilon_{a b} \xi+\chi_{a b},
\end{aligned}
$$

where $\chi_{a b}$ is anti-symmetric and trace free, $\epsilon^{a b} \chi_{a b}=0$. The reality condition on $8_{s}$ thus imposes the 'self-duality' condition

$$
\chi_{a b}^{*}=\epsilon^{a c} \epsilon^{b d} \chi_{c d} .
$$

on $\chi_{a b}$ (the hermitian metric is implicit in this equation).

The relation between the three covariantly constant spinors $\eta_{r}, r=1,2,3$, normalized such that $\eta_{r}^{T} \eta_{r}=1$, and the three covariantly constant Kähler forms on a hyper-Kähler manifold is

$$
\Omega_{i j}^{r}=\epsilon^{r s t} \eta_{s}^{T} \gamma_{i j} \eta_{t} / 2
$$

where the $\gamma_{i}$ are $S O(8)$ gamma matrices. This is also reflected in the branching of the $\mathbf{2 8}$ exhibited above, which contains three singlets from the $(\mathbf{1}, \mathbf{3})$ when the holonomy is $S p(2)$. 
A general two-form on a quaternionic Kähler manifold, say the curvature of a connection $A_{a A}$, has the form $F_{a A b B}$. Decomposing this into its irreducible pieces according to

$$
\begin{aligned}
\mathbf{2 8} & =\mathbf{8}_{\mathbf{v}} \wedge 8_{\mathrm{v}} \rightarrow(4,2) \wedge(4,2) \\
& =(4 \wedge 4,2 \subseteq 2)+(4(5), 2 \wedge 2) \\
& =(1,3)+(5,3)+(10,1),
\end{aligned}
$$

one obtains (in the notation of [15])

$$
F_{a A b B}=\epsilon_{a b} G_{A B}+K_{a b A B}+\epsilon_{A B} H_{a b}
$$

where $G$ and $H$ are symmetric and

$$
K_{a b A B}=\frac{1}{2}\left(F_{a A b B}+F_{a B b A}\right)-\epsilon_{a b} G_{A B}
$$

is symmetric in $(A B)$ and skew trace-free in $(a b)$.

In 15], Ward discusses $S p(2) \times S p(1)$ invariant integrable equations for gauge fields, and he obtains the set of 18 equations

$$
G_{A B}=K_{a b A B}=0
$$

as the integrability conditions $\pi^{A} \pi^{B} F_{a A b B}=0$ for the equations $\pi^{A} D_{a A} \psi=$ 0 . On a hyper-Kähler manifold, one can split these equations invariantly into three parts and consider one of them seperately, say

$$
G_{12}+G_{21}=K_{a b 12}+K_{a b 21}=0 .
$$

It is this set of $(5+1)$ equations that one obtains from SYM theory. Indeed, the field content is (displaying also the $S O(1,1)$ R-symmetry quantum numbers)

$$
\begin{aligned}
& 10 \rightarrow 2 \times 4^{0}+1^{+2,-2} \\
& 16 \rightarrow 5^{-1}+3 \times 1^{-1}+2 \times 4^{+1}
\end{aligned}
$$

Here we recognise, first of all, the gauge field $A_{a A}=\left(A_{a 1}, A_{a 2}\right)$ and its ghost-number one superpartner $\psi_{a A}$. Secondly, one sees the two scalars, say $\eta_{11}$ and $\eta_{22}$ required to gauge fix the $\psi_{a A}$. And thirdly there are the antighosts $\eta$ and $\chi_{a b}$ imposing the self-duality conditions (3.22). Finally, as in Donaldson theory there are a bosonic anti-ghost and a ghost-for-ghost. The theory has has the unusal property of possessing an $N_{T}=3$ symmetry, all supercharges carrying the same $S O(1,1)$ ghost number and tranforming as an $S p(1)$ triplet. 
These equations are the reduction under $S U(4) \rightarrow S p(2)$ of the holomorphic self-duality equations of the $S U(4)$ H-theory [1] discussed above (by an appropriate choice of complex structure). As such, thinking of the latter as a holomoprhic version of Donaldson theory, the hyper-Kähler equations may play a role akin to that of instanton equations on symplectic or Kähler four-manifolds.

\section{A Notation}

Our conventions for $\gamma$-matrices in $d=4$ and $d=(5+1)$ are the following: For $d=4$ the gamma matrices are taken to be hermitian and in terms of Pauli matrices $\sigma_{a}$ we choose

$$
\begin{aligned}
& \gamma_{a}=\sigma_{1} \otimes \sigma_{a} \\
& \gamma_{4}=\sigma_{2} \otimes \mathbb{I},
\end{aligned}
$$

so that $\gamma_{5}$ is diagonal,

$$
\gamma_{5}=\sigma_{3} \otimes \mathbb{I}
$$

The 'charge conjugation' matrix $C=\gamma_{2} \gamma_{4}$ satisfies $C^{T}=-C$ and

$$
C \gamma_{m}=\gamma_{m}^{T} C
$$

where $m=1, \ldots, 5$.

The conjugate of a spinor is

$$
\bar{\Psi}=\Psi^{* T}
$$

while the chiral decomposition is

$$
\Psi=\left(\begin{array}{c}
\psi_{+} \\
\psi_{-}
\end{array}\right)
$$

For $d=(5+1)$ Minkowski space we choose

$$
\begin{aligned}
\Gamma^{m} & =\sigma_{1} \otimes \gamma_{m} \\
\Gamma^{0} & =i \sigma_{2} \otimes \mathbb{I}_{4}
\end{aligned}
$$

so that $\Gamma^{7}$ is also diagonal,

$$
\Gamma^{7}=\sigma_{3} \otimes \mathbb{I}_{4}
$$


Lorentz generators are denoted by

$$
\Sigma^{M N}=\frac{1}{4}\left[\Gamma^{M}, \Gamma^{N}\right] .
$$

The charge conjugation matrix $\mathcal{C}$ satisfying

$$
\mathcal{C} \Gamma_{m}=\Gamma_{m}^{T} \mathcal{C}, \quad \mathcal{C} \Gamma_{0}=-\Gamma_{0}^{T} \mathcal{C}
$$

as well as

$$
\mathcal{C}^{T}=-\mathcal{C}=\mathcal{C}^{-1}
$$

is

$$
\mathcal{C}=\mathbb{I}_{2} \otimes C
$$

\section{REFERENCES}

[1] B. Zumino, Euclidean supersymmetry and the many-instanton problem, Phys. Lett. 69B, (1977) 369-371.

[2] P. van Nieuwenhuizen, A. Waldron On Euclidean spinors and Wick rotations, Phys. Lett. B389 (1996) 29-36, hep-th/9608174; A continuous Wick rotation for spinor fields and supersymemtry in Euclidean space, hep-th/9611043.

[3] E. Witten, Topological quantum field theory, Commun. Math. Phys. 117 (1988) 353.

[4] J. Yamron, Topological actions from twisted supersymmetric theories, Phys. Lett. B213 (1988) 325-330.

[5] C. Vafa, E. Witten, A strong coupling test of S-duality, Nucl. Phys. B431 (1994) 3-77, hep-th/9408074.

[6] N. Marcus, The other topological twisting of $N=4$ Yang-Mills, Nucl. Phys. B452 (1995) 331, hep-th/9506002.

[7] M. Bershadsky, V. Sadov, C. Vafa, D-branes and topological field theories, Nucl. Phys. B463 (1996) 420, hep-th/9511222.

[8] M. Blau, G. Thompson, Aspects of $N_{T} \geq 2$ topological gauge theories and D-branes, Nucl. Phys. B492 (1997) 545-590, hep-th/9612143.

[9] J.M.F. Labastida, C. Lozano, Mathai-Quillen formulation of twisted $N=4$ supersymmetric gauge theories in four dimensions, hep-th/9702106.

[10] E. Witten, Bound states of strings and p-branes, Nucl. Phys. B460 (1996) 335. hep-th/9510135.

[11] L Baulieu, H. Kanno, I.M. Singer, Special quantum field theories in eight and other dimensions, hep-th/9704167; Cohomological Yang-Mills theory in eight dimensions, hep-th/9705127. 
[12] B.S. Acharya, M. O'Loughlin, B. Spence, Higher diemnsional analogues of Donaldson-Witten theory, hep-th/9705138.

[13] B.S. Acharya, M. O'Loughlin, Self-duality in $D \leq 8$-dimensional Euclidean gravity, hep-th/9612182.

[14] E. Corrigan, C. Devchand, D. Fairlie, J. Nuyts, First-order equations for gauge fields in spaces of dimension greater than four, Nucl. Phys. B214 (1983) 452464.

[15] R.S. Ward, Completely solvable gauge-field equations in dimensions greater than four, Nucl. Phys. B236 (1984) 381-396.

[16] S.K. Donaldson, R.P. Thomas, Gauge theory in higher dimensions, Oxford preprint

[17] T. Kugo, P. Townsend, Supersymmetry and the division algebras, Nucl. Phys. B221 (1983) 357-380.

[18] M. Bershadsky, A. Johansen, V. Sadov, C. Vafa, Topological reduction of $4 d$ SYM to 2d $\sigma$-models, Nucl. Phys. B448 (1995) 166, hep-th/9501096.

[19] N. Seiberg, Notes on theories with 16 supercharges, hep-th/9705117.

[20] M. Blau, G. Thompson, $N=2$ topological gauge theory, the Euler characteristic of moduli spaces, and the Casson invariant, Commun. Math. Phys. 152 (1993) 41-71, hep-th/9112012.

[21] R. Dijkgraaf, G. Moore, Balanced topological field theories, Commun. Math. Phys. 185 (1997) 411-440, hep-th/9608169.

[22] R. Bryant, Classical, exceptional, and exotic holonomies: a status report, in Actes de la Table Ronde de Géométrie Différentielle en l'Honneur de Marcel Berger, Collection SMF Séminaires and Congrès 1 (1996) (Soc. Math. de France), 93-166, available as http://www .math.duke.edu/preprints/95-10.dvi.

[23] A. Losev, G. Moore, N. Nekrasov, S. Shatashvili, Chiral Lagrangians, anomalies, supersymmetry, and holomorphy, Nucl. Phys. B484 (1997) 196-222, hep-th/9606082. 